\title{
Accuracy of radiotherapy dose calculations based on cone-beam CT: comparison of deformable registration and image correction based methods
}

DOI:

10.1088/1361-6560/aabOf0

\section{Document Version}

Accepted author manuscript

Link to publication record in Manchester Research Explorer

Citation for published version (APA):

Marchant, T., D Joshi, K., \& Moore, C. (2018). Accuracy of radiotherapy dose calculations based on cone-beam CT: comparison of deformable registration and image correction based methods. Physics in Medicine and Biology, 63, [065003]. https://doi.org/10.1088/1361-6560/aabof0

Published in:

Physics in Medicine and Biology

\section{Citing this paper}

Please note that where the full-text provided on Manchester Research Explorer is the Author Accepted Manuscript or Proof version this may differ from the final Published version. If citing, it is advised that you check and use the publisher's definitive version.

\section{General rights}

Copyright and moral rights for the publications made accessible in the Research Explorer are retained by the authors and/or other copyright owners and it is a condition of accessing publications that users recognise and abide by the legal requirements associated with these rights.

\section{Takedown policy}

If you believe that this document breaches copyright please refer to the University of Manchester's Takedown Procedures [http://man.ac.uk/04Y6Bo] or contact uml.scholarlycommunications@manchester.ac.uk providing relevant details, so we can investigate your claim.

\section{OPEN ACCESS}


ACCEPTED MANUSCRIPT

\section{Accuracy of radiotherapy dose calculations based on cone-beam CT: comparison of deformable registration and image correction based methods}

To cite this article before publication: Tom Edward Marchant et al 2018 Phys. Med. Biol. in press https://doi.org/10.1088/1361-6560/aab0f0

\section{Manuscript version: Accepted Manuscript}

Accepted Manuscript is "the version of the article accepted for publication including all changes made as a result of the peer review process, and which may also include the addition to the article by IOP Publishing of a header, an article ID, a cover sheet and/or an 'Accepted

Manuscript' watermark, but excluding any other editing, typesetting or other changes made by IOP Publishing and/or its licensors"

This Accepted Manuscript is (C) 2018 Institute of Physics and Engineering in Medicine.

During the embargo period (the 12 month period from the publication of the Version of Record of this article), the Accepted Manuscript is fully protected by copyright and cannot be reused or reposted elsewhere.

As the Version of Record of this article is going to be / has been published on a subscription basis, this Accepted Manuscript is available for reuse under a CC BY-NC-ND 3.0 licence after the 12 month embargo period.

After the embargo period, everyone is permitted to use copy and redistribute this article for non-commercial purposes only, provided that they adhere to all the terms of the licence https://creativecommons.org/licences/by-nc-nd/3.0

Although reasonable endeavours have been taken to obtain all necessary permissions from third parties to include their copyrighted content within this article, their full citation and copyright line may not be present in this Accepted Manuscript version. Before using any content from this article, please refer to the Version of Record on IOPscience once published for full citation and copyright details, as permissions will likely be required. All third party content is fully copyright protected, unless specifically stated otherwise in the figure caption in the Version of Record.

View the article online for updates and enhancements. 


\title{
Accuracy of radiotherapy dose calculations based on cone-beam CT: comparison of deformable registration and image correction based methods
}

\author{
T E Marchant ${ }^{1,2}$, K D Joshi ${ }^{2}$ and C J Moore ${ }^{1}$ \\ ${ }^{1}$ The University of Manchester, Manchester Academic Health Science Centre, The Christie \\ NHS Foundation Trust, Manchester, M20 4BX, UK. \\ ${ }^{2}$ Christie Medical Physics and Engineering, The Christie NHS Foundation Trust, Manchester, \\ M20 4BX, UK. \\ E-mail: tom.marchantechristie.nhs.uk
}

\begin{abstract}
Radiotherapy dose calculations based on cone-beam CT (CBCT) images can be inaccurate due to unreliable Hounsfield units (HU) in the CBCT. Deformable image registration of planning $\mathrm{CT}$ images to $\mathrm{CBCT}$, and direct correction of $\mathrm{CBCT}$ image values are two methods proposed to allow heterogeneity corrected dose calculations based on CBCT. In this paper we compare the accuracy and robustness of these two approaches. CBCT images for 44 patients were used including pelvis, lung and head \& neck sites. CBCT HU were corrected using a "shading correction" algorithm and via deformable registration of planning CT to CBCT using either Elastix or Niftyreg. Radiotherapy dose distributions were recalculated with heterogeneity correction based on the corrected CBCT and several relevant dose metrics for target and OAR volumes were calculated. Accuracy of CBCT based dose metrics was determined using an "override ratio" method where the ratio of the dose metric to that calculated on a bulk-density assigned version of the same image is assumed to be constant for each patient, allowing comparison to the patient's planning CT as a gold standard. Similar performance is achieved by shading corrected CBCT and both deformable registration algorithms, with mean and standard deviation of dose metric error less than $1 \%$ for all sites studied. For lung images, use of deformed CT leads to slightly larger standard deviation of dose metric error than shading corrected CBCT with more dose metric errors greater than $2 \%$ observed ( $7 \%$ vs $1 \%)$.
\end{abstract}

Keywords: Cone-beam CT, Image-guided radiotherapy, Deformable image registration.

\section{Introduction}

Cone beam CT (CBCT) imaging is commonly used in radiotherapy (RT) for verification of patient and target position (Jaffray et al 2002, McBain et al 2006). Use of CBCT imaging can improve the quality of RT by reducing geometric errors, leading to the use of smaller margins, and providing potential for adaptive RT. However CBCT images often have poorly calibrated Hounsfield Units (HU) and are subject to artefacts and non-uniformity due to X-ray scatter, patient motion and detector persistence effects (Siewerdsen and Jaffray 2001, Marchant et al 


\section{CBCT dose calculation accuracy comparison}

2011, Mail et al 2008). As a result, tissue densities from CBCT are not suitable for use in heterogeneity-corrected re-calculation of RT delivered dose.

Several methods have been introduced to enable attenuation corrected RT dose calculations based on CBCT images. These include calibration of CBCT using HU-density curves derived from contrast phantom scans (Yang et al 2007, Guan and Dong 2009) or patient images (Fotina et al 2012, Richter et al 2008), scatter corrections (Zhu et al 2009, Thing et al 2016, Dunlop et al 2015), uniformity corrections incorporating prior-information either in projection space (Niu et al 2010) or reconstructed image space (Fan et al 2015, Marchant et al 2008), and deformable image registration (DIR) with planning CT (pCT) (Veiga et al 2015, Moteabbed et al 2015).

Calibration of CBCT using a calibration curve derived from contrast phantom scans has been proposed (Yang et al 2007, Guan and Dong 2009). Studies have been based on phantom images or a small number of patient images and have reported variable accuracy of dose calculations based on calibrated CBCT. Yang et al (2007) reported good dose calculation accuracy $(<2 \%)$ for 3 prostate cases, but larger errors in 1 lung case (up to $5 \%$ ). Guan and Dong (2009) found CBCT-based dose calculation errors in a pelvic phantom of $2.5 \%-6.7 \%$ depending on the planning technique used.

CBCT calibration curves derived from regions of different tissue types in clinical images have also been proposed. Richter et al (2008) found dose errors less than $5 \%$ using either patient specific or group-based calibration. Fotina et al (2012) reported a large spread of dose values using population based calibration. However both of these studies assessed accuracy of CBCT dose calculations by comparison to the planning CT dose distribution. Therefore they do not account for changes in anatomy between CT and CBCT acquisition.

Zhu et al (2009) and Thing et al (2016) proposed CBCT scatter correction methods, but did not evaluate the effect on radiotherapy dose calculation accuracy. Dunlop et al (2015) found that dose calculations based on scatter corrected CBCT were acceptable for H\&N images (errors up to $1.7 \%$ ) but less so for pelvis (errors up to $4.2 \%$ ) and lung (errors up to $8.0 \%)$.

In DIR-based methods the planning CT is deformed to match the CBCT anatomy, allowing it to be used as the patient attenuation map for radiotherapy dose calculations. Use of DIR for CBCT-based dose assessment of RT is gaining popularity, with a number of commercial products recently incorporating this workflow. Studies of dose calculation accuracy using deformed CT have shown promising results, although based on small numbers of test images. Veiga et al (2015) used images from 5 head and neck (H\&N) patients and found dose errors generally less than $2 \%$. Moteabbed et al (2015) found small errors $(<1 \%)$ in DIR-based CBCT dose calculations using images of a pelvic phantom. Detailed studies of CBCT-based dose calculation accuracy based on larger numbers of clinical images for a range of anatomical sites are still required.

We previously developed a prior-image based correction method to restore HU values and improve uniformity of CBCT images (Marchant et al 2008) (referred to here as the "shading correction"), which allows RT dose calculations based on corrected CBCT accurate to within 1-2 \% (Marchant et al 2017). 


\section{CBCT dose calculation accuracy comparison}

In this paper we compare the accuracy of CBCT-based RT dose assessment using either shading corrected CBCT images or deformable image registration. Our comparison includes two different open-source deformable image registration packages and is based on a large sample of clinical CBCT images at three different anatomical sites. Clinical images are used for the evaluation, rather than phantom images, in order to evaluate the effect of anatomical changes that occur between planning CT and CBCT acquisition, for example weight loss, development of atelectasis in the lung or changes in rectum/bladder filling. These changes may be quite large and have the potential to reduce the effectiveness of DIR. The shading correction uses prior information from the planning CT so it is also important to test its robustness to anatomical changes in the CBCT. We also introduce a method to assess the accuracy of RT dose metrics derived from CBCT dose calculations based on comparison to a density overridden version of the same image (see section section 2.4.3).

\section{Methods}

\subsection{Cone beam CT images}

CBCT images and RT plan data were collected for 44 patients undergoing RT (15 pelvis, $14 \mathrm{H} \& \mathrm{~N}, 15$ lung). Three CBCT images per patient were included in the study (132 CBCT images total). CBCT images were acquired by the Elekta XVI linac-integrated CBCT system (Elekta AB, Stockholm, Sweden), using XVI versions 4.5 or 5.0. The study was performed retrostpectively using only images acquired routinely as part of the patients' RT. All image and RT plan data was anonymized and ethical approval was obtained for inclusion in the study (UK REC ref. 15/LO/0146).

\subsection{Shading correction algorithm}

A previously reported "shading correction" algorithm was used to process the CBCT images to improve HU calibration and remove non-uniformity artifacts (Marchant et al 2008). The algorithm uses the patient's planning CT image as prior information. Image histograms of the CBCT and pCT images are compared and a linear scaling is derived which best matches the main histogram peak positions (air and soft tissue) between the CBCT and pCT images (Joshi and Marchant 2017). Next regions representing soft-tissue are identified in both images and other regions (e.g. air, lung, bone) are removed and filled by interpolation from surrounding soft-tissue areas. The CBCT image is then divided by the rigidly registered pCT image to create a "shading map" containing the relative brightness of each pixel in the CBCT image compared to the pCT. The shading map is smoothed so that it retains only low spatial frequency variations in brightness, and is then applied as a correction map for the CBCT image to produce the final corrected image. An example pelvis CBCT image is shown in Figure 1 before and after applying the shading correction. 


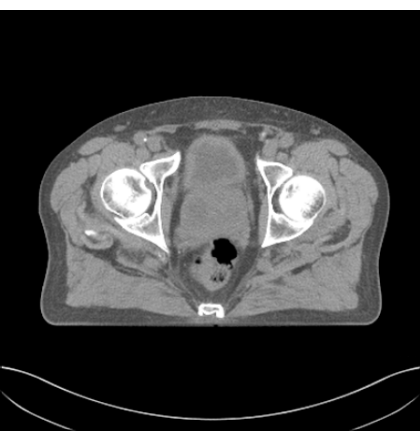

CT

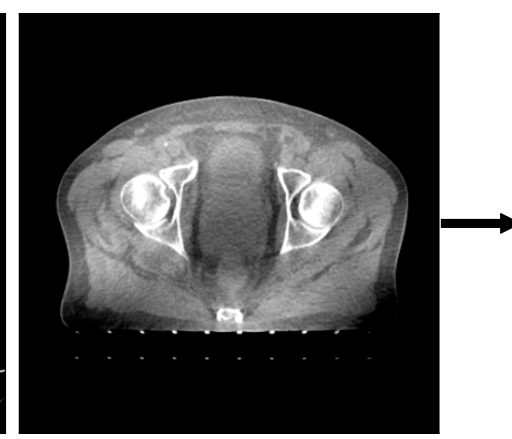

CBCT

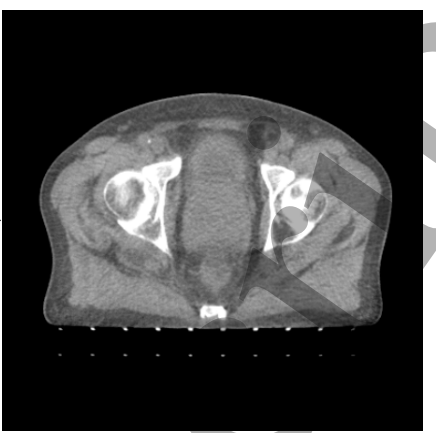

Corrected CBCT

Figure 1. Pelvis $\mathrm{CT}$ and $\mathrm{CBCT}$ images before and after application of the shading correction (all displayed with window level 0, width 500).

\subsection{Deformable image registration}

Deformable image registration was used to deform each patient's pCT to match the CBCT images for the same patient. DIR was done using two software packages: Elastix and Niftyreg. The algorithm parameters selected for each package were based on ones used in previous studies (see below) and while not systematically optimised with our data they were observed to produce good results in most of the test images.

\subsubsection{Elastix Elastix (Klein et al 2010) is an open-source software package for rigid and} non-rigid registration of medical images (http://elastix.isi.uu.nl). Elastix has previously been used for registration bewteen CT and CBCT images for lung (Thing et al 2016), prostate (Kim et al 2013) and H\&N sites (Nithiananthan et al 2011). Parameters for Elastix deformable registration were based on examples for intra-patient CT image registration available on the Elastix parameter file database (http://elastix.bigr.nl/wiki/index.php/Parameter_file_database). DIR with Elastix used normalized mutual information similarity metric with a B-spline parameterized transformation. A five-level multiresolution registration scheme was used with image resolution and grid spacing downsampled by a factor of 2 at each multiresolution level, and final B-spline grid spacing of $15 \mathrm{~mm}$. Gradient descent optimisation was used with up to 1000 iterations at each multiresolution level.

2.3.2. Niftyreg NiftyReg (http://cmictig.cs.ucl.ac.uk/research/software/software-nifty) is an open source image registration package implemented by the Centre of Medical Image Computing at University College London, which has previously been applied for RT dose calculations based on deformed CT in head and neck sites (Veiga et al 2014). An initial rigid registration of $\mathrm{pCT}$ to $\mathrm{CBCT}$ was followed by DIR using the B-spline Free Form Deformation algorithm (Rueckert et al 1999, Modat et al 2010). The localized normalized cross-correlation (LNCC) similarity measure was used as this is able to account for spatial variation of CBCT pixel values by calculating similarity at each point over a local window (5 $\mathrm{mm}$ window size) (Veiga et al 2015). 


\section{CBCT dose calculation accuracy comparison}

\subsection{Assessment of dose calculation accuracy}

2.4.1. Phantom study CT and CBCT images of the Alderson Rando phantom (RSD Inc, Long Beach, CA, USA) were used to test the accuracy of CBCT-based dose calculations. A five-field prostate IMRT plan and a Lung SBRT VMAT plan were created using using the Pinnacle RT planning system (v9.8, Philips Radiation Oncology Systems, Fitchburg, WI, USA) using representative target and organ at risk (OAR) volumes. Each plan was then copied onto co-registered CBCT images of the phantom before and after applying the shading correction. All beam parameters (including monitor units) were keptidentical to the original plan. The dose was recalculated using heterogeneity correction based on the CBCT image values. Several dose metrics were evaluated for each CBCT dose calculation and compared to the original plan. The dose metrics compared were mean dose to planning target volume (PTV), dose covering $95 \%$ of PTV, volume of rectum receiving greater than $40 \mathrm{~Gy}$ (prostate plan only) and maximum dose to spinal cord (lung plan only). Since the phantom does not deform between CT and CBCT images, the dose metric calculated from CBCT can be directly compared to the originally planned value.

\subsubsection{Patient image study All patient images (CBCT, corrected CBCT, and deformed CT)} were imported to the Pinnacle RT planning system. RT dose distributions were re-calculated based on each patient's original RT plan and using CBCT image values for heterogeneity correction. Here CBCT could be either the raw CBCT, corrected CBCT, or deformed CT image. Table 1 shows further details of the treatment sites and techniques included in the study. CBCT images were included in the study only if the field of view (FOV) was sufficient to include the patient outline at all points where it is crossed by the RT beams. This ensures that RT dose can be calculated in all relevant areas. Most images acquired with Medium or Large FOV (41 cm or $50 \mathrm{~cm}$ diameter) satisfy this requirement. H\&N patients are usually imaged with Small FOV $(26 \mathrm{~cm})$ which does not encompass the patient outline below the shoulders. Hence dose cannot be calculated based on the CBCT image in this region, without use of other methods to reconstruct the missing regions of the patient. For H\&N patients in this study we evaluated only dose to the primary disease target volume, and did not consider dose to any nodal target volume that extended below the level of the shoulders. 
Table 1: Radiotherapy plan characteristics for validation of CBCT based dose calculations. Abbreviations: 3D conformal radiotherapy (3DCRT), Volumetric modulated arc therapy (VMAT), Intensity modulated radiotherapy (IMRT), Stereotactic ablative body radiotherapy (SABR).

For each plan three clinically relevant dose metrics were calculated: mean dose to PTV, Dose covering $95 \%$ of PTV $\left(\mathrm{D}_{95}\right)$, and an OAR dose metric relevant to the target site: volume of rectum receiving greater than $40 \mathrm{~Gy}\left(\mathrm{~V}_{40 \mathrm{~Gy}}\right)$ for pelvis plans, maximum dose to spinal cord (SC) for lung plans, and contralateral parotid mean dose for $\mathrm{H} \& \mathrm{~N}$ plans. Pelvis and lung CBCT dose metrics were calculated based on PTV and OAR contours that had been propogated rigidly from $\mathrm{pCT}$ and then manually edited to account for any large changes between the images (e.g. to avoid contours extending beyond patient surface or into bone/air cavities). For H\&N images the PTV and OAR contours were more commonly situated close to bone or the patient surface so were propogated from CT via DIR and visually inspected for anatomical plausibility.

2.4.3. Override ratio validation method For patient images there will be changes between CT and CBCT imaging, so the doses calculated from CBCT are not expected to be the same as the originally planned values. A method to test accuracy of CBCT dose calculations by comparison to a density overridden version of the same scan was developed and validated. This method was previously used in (Marchant et al 2017) but is described here for completeness.

Because of anatomical changes between the pCT and CBCT images, it is expected that the dose metric calculated on CBCT will not be identical to that calculated on pCT. Instead the dose calculation is repeated after applying bulk density overrides to the image, and the ratio of the dose metric before and after applying the density override is calculated (the "override ratio"). It is expected that this ratio will remain constant for a particular patient and RT plan. This is because the error introduced by applying the density override depends mainly on the individual patient's tissue densities and each beam's path length through different tissues. These factors remain approximately constant for each patient/plan. Therefore the error in CBCT-based dose metrics can be revealed by comparing the CBCT override-ratio for each metric to that calculated from the pCT image (the gold standard). The density overrides that 
were applied varied for each anatomical site. For pelvis images all tissue within the body contour was assigned density of $1 \mathrm{gcm}^{-3}$. For lung images the lung tissue was also assigned a uniform density based on that patient's mean lung HU. For H\&N images, overrídes were applied for soft-tissue, bone and air cavities within the patient.

Figure 2 illustrates the procedure, where the override ratio for a dose metric calculated from CBCT, $O R_{C B C T}=(C / D)$, should be the same as that calculated from pCT, $O R_{C T}=$ $(A / B)$. Any difference between the override ratio for $\mathrm{CT}$ and $\mathrm{CBCT}$ is attributed to the error in the CBCT dose calculation. Therefore the CBCT dose metric relative error is $\left(O R_{C B C T} / O R_{C T}\right)-1$.

The override-ratio method for determining accuracy of CBCT dose calculations was validated using 5 patients with repeat pCT (4 lung, 1 pelvis case). The override-ratio was calculated for target and OAR dose metrics for both $\mathrm{pCT}$ images for each patient, using the patient's original treatment plan. The assumption that the override-ratio remains constant for a particular patient, RT plan and dose metric could then be tested. Patients having repeat $\mathrm{pCT}$ tend to be those where large anatomical changes occur during RT. Hence this sample, while small, serves to test robustness of the override-ratio method to large changes between images.

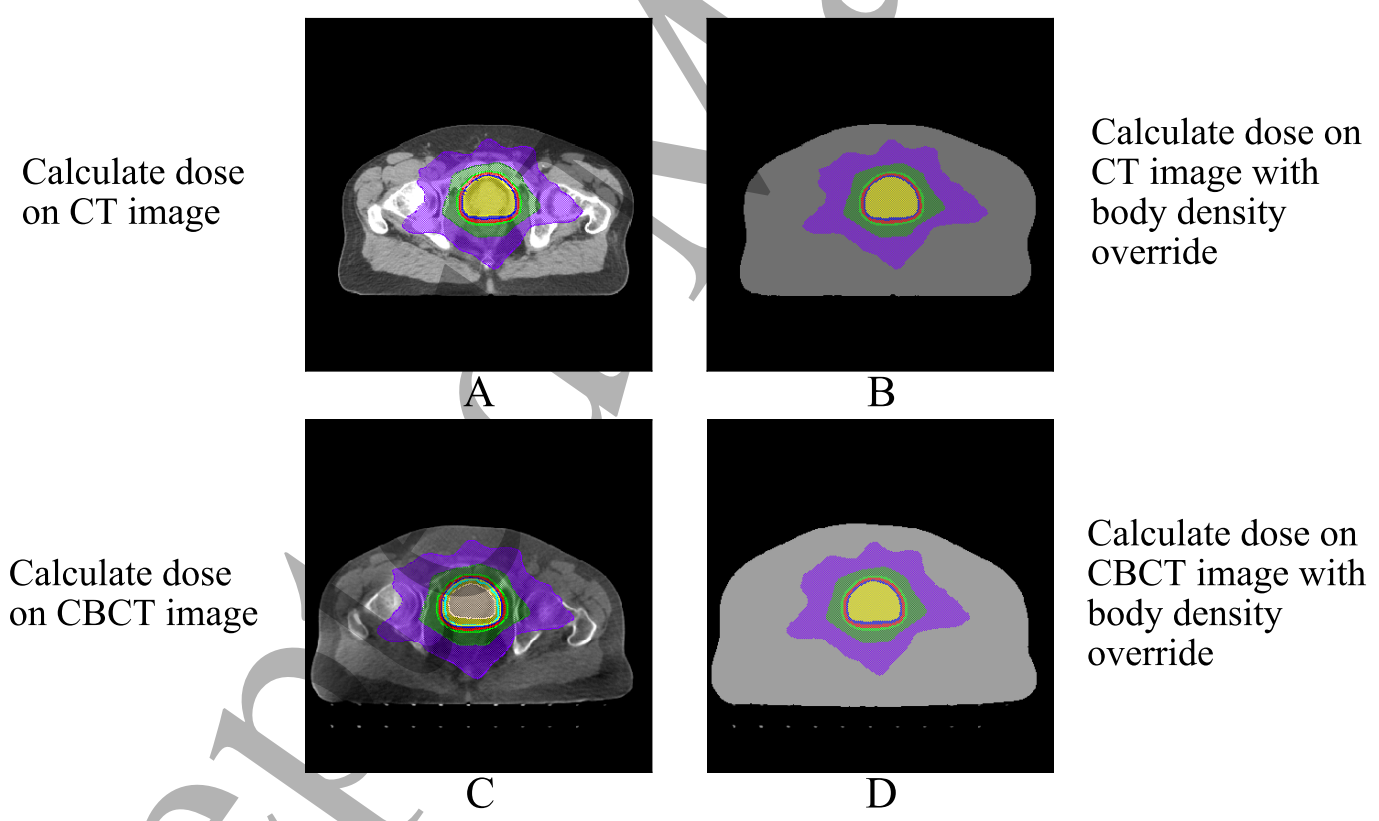

Figure 2. Override ratio method to determine errors in dose metrics calculated from CBCT.

\section{Results}

\subsection{Phantom study}

Figure 3 shows protate plan dose distributions calculated for planning CT, uncorrected CBCT, and shading corrected CBCT. Table 2 shows the PTV and rectum dose metrics calculated using each image, along with the error relative to planning $\mathrm{CT}$ for each of the $\mathrm{CBCT}$ images. 
Uncorrected CBCT results in large dose errors $(+4.7 \%$ for PTV, $+24 \%$ for rectum V40Gy). After shading correction all dose metric errors are much smaller $(<2 \%)$.
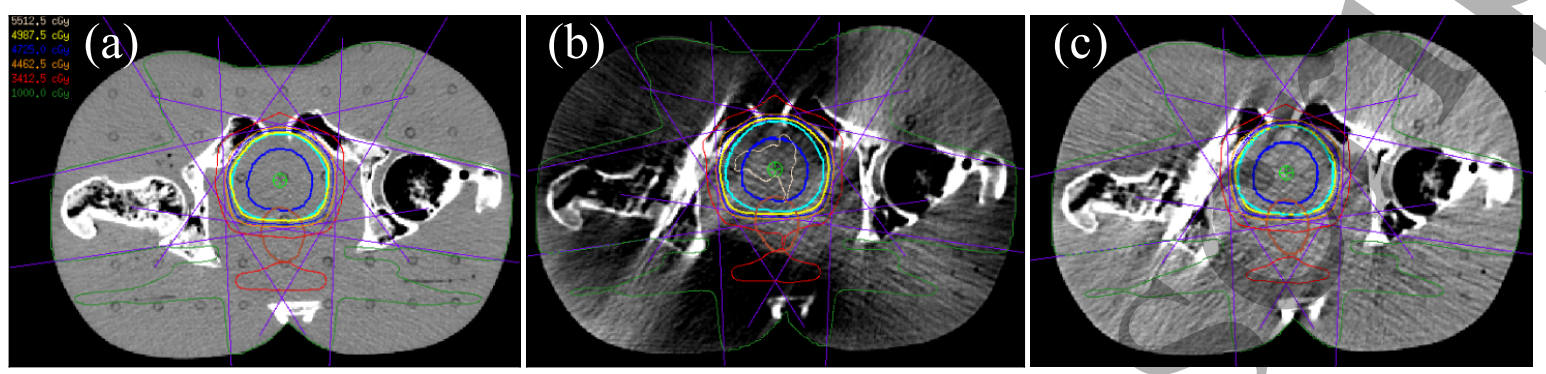

Figure 3. Prostate IMRT plan dose distributions calculated on (a) CT, (b) CBCT raw, and (c) shading corrected CBCT. All images are displayed with the same window and level settings.

Table 2: Prostate IMRT plan dose metric errors.

\begin{tabular}{cccc}
\hline Image & PTV mean & PTV D95 & Rectum V40Gy \\
\hline CT & $5199 \mathrm{cGy}$ & $5091 \mathrm{cGy}$ & $27.8 \mathrm{cc}$ \\
CBCT raw & $5441 \mathrm{cGy}(+4.7 \%)$ & $5330 \mathrm{cGy}(+4.7 \%)$ & $34.5 \mathrm{cc}(+24.1 \%)$ \\
CBCT shaded & $5186 \mathrm{cGy}(-0.3 \%)$ & $5083 \mathrm{cGy}(-0.2 \%)$ & $27.4 \mathrm{cc}(-1.4 \%)$ \\
\hline
\end{tabular}

Figure 4 shows lung plan dose distributions calculated for planning CT, uncorrected CBCT, and shading corrected CBCT. Table 3 shows the PTV and spinal cord dose metrics calculated using each image, along with the error relative to planning $\mathrm{CT}$ for each of the CBCT images. Uncorrected CBCT results in dose errors greater than $2 \%$ for dose covering $95 \%$ of PTV (D95) and spinal cord max dose. After shading correction all dose metric errors are reduced and are less than $2 \%$.
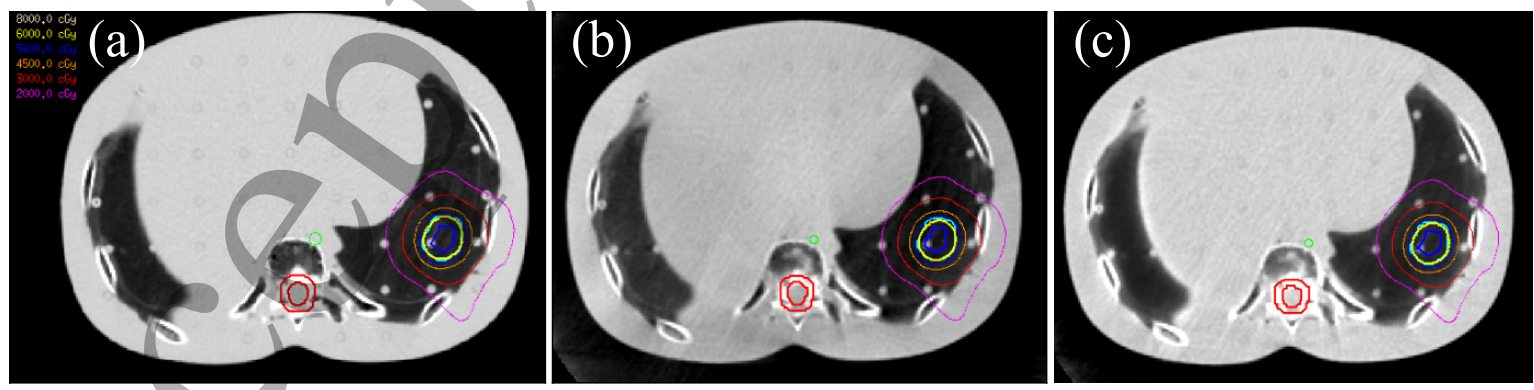

Figure 4. Lung SABR plan dose distributions calculated on (a) CT, (b) CBCT raw, and (c) shading corrected CBCT. All images are displayed with the same window and level settings. 
Table 3: Lung SABR plan dose metric errors.

\begin{tabular}{cccc}
\hline Image & PTV mean & PTV D95 & SC $\max (1 \mathrm{cc})$ \\
\hline CT & $6423 \mathrm{cGy}$ & $6001 \mathrm{cGy}$ & $590 \mathrm{cGy}$ \\
CBCT raw & $6347 \mathrm{cGy}(-1.2 \%)$ & $5829 \mathrm{cGy}(-2.9 \%)$ & $615 \mathrm{cGy}(+4.2 \%)$ \\
CBCT shaded & $6399 \mathrm{cGy}(-0.4 \%)$ & $5925 \mathrm{cGy}(-1.3 \%)$ & $588 \mathrm{cGy}(-0.3 \%)$ \\
\hline
\end{tabular}

\subsection{Validation of override-ratio method}

For the five cases with repeat pCT, the differences between override-ratios calculated from the two CT images were small. Mean difference was $0.5 \%$ with standard deviation $0.7 \%$ (range -1.7 to $+0.6 \%$ ). Figure 5 a shows the distribution of differences in override-ratio between the two CT images. It should be noted that this validation included some cases showing quite large anatomical changes between the two pCT images. Figure $5 \mathrm{~b}-\mathrm{c}$ shows original and repeat pCT images for one of the lung cases with large change in lung volume and tumour size. Because of anatomical changes, the dose metrics tested were not always similar between the two images. Four out of five patients had at least one metric which differed by $>3 \%$ between the two images, and a maximum difference of $39 \%$ was observed. The presence of these differences shows that override-ratio values obtained for two images of the same patient are consistent even if there are considerable anatomical changes between the images.

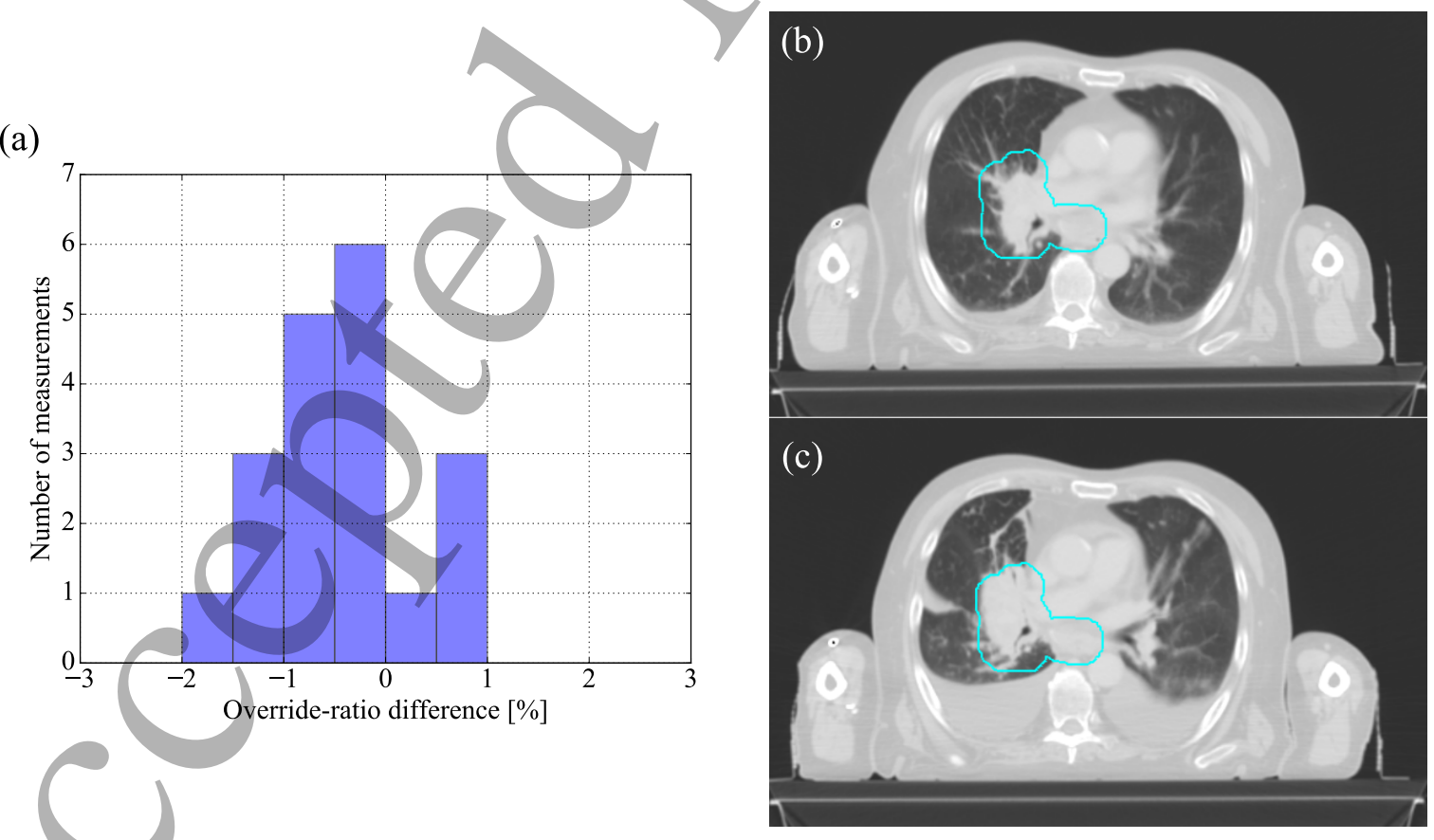

Figure 5. (a) Histogram showing differences between override-ratios calculated for two CT images of the same patient. ( $\mathrm{b} \& \mathrm{c}$ ) Lung case showing large change between original (b) and repeat (c) pCT images. Radiotherapy planning target volume (PTV) is shown in light blue. 


\section{CBCT dose calculation accuracy comparison}

\subsection{Deformable image registration performance}

Both DIR algorithms performed well for the majority of images. Body surface and bone surfaces were usually well matched between deformed CT and CBCT. Soft tissue details were also well matched in regions where deformations were small or smooth. However, neither Elastix or Niftyreg was able to accurately reproduce large or complex changes such as large change in bladder filling, movement of small bowel loops or appearance/disappearance of gas pockets in the rectum. Figure 6 shows an example slice from a pelvis image where soft tissue deformations around the rectum and bladder are not accurately reproduced in the deformed CT. White arrows indicate regions where the deformed CT does not match the CBCT. Figure 7 shows example lung images for a case where the lung contour has changed markedly on the CBCT due to atelectasis. Neither DIR algorithm is able to reproduce this change, with Elastix (figure 7b) matching the reduced lung volume but introducing large deformations to the surrounding spine and rib bones, and Niftyreg (figure $7 \mathrm{~d}$ ) introducing less distortion to the surrounding anatomy but failing to accurately represent the reduced lung volume.

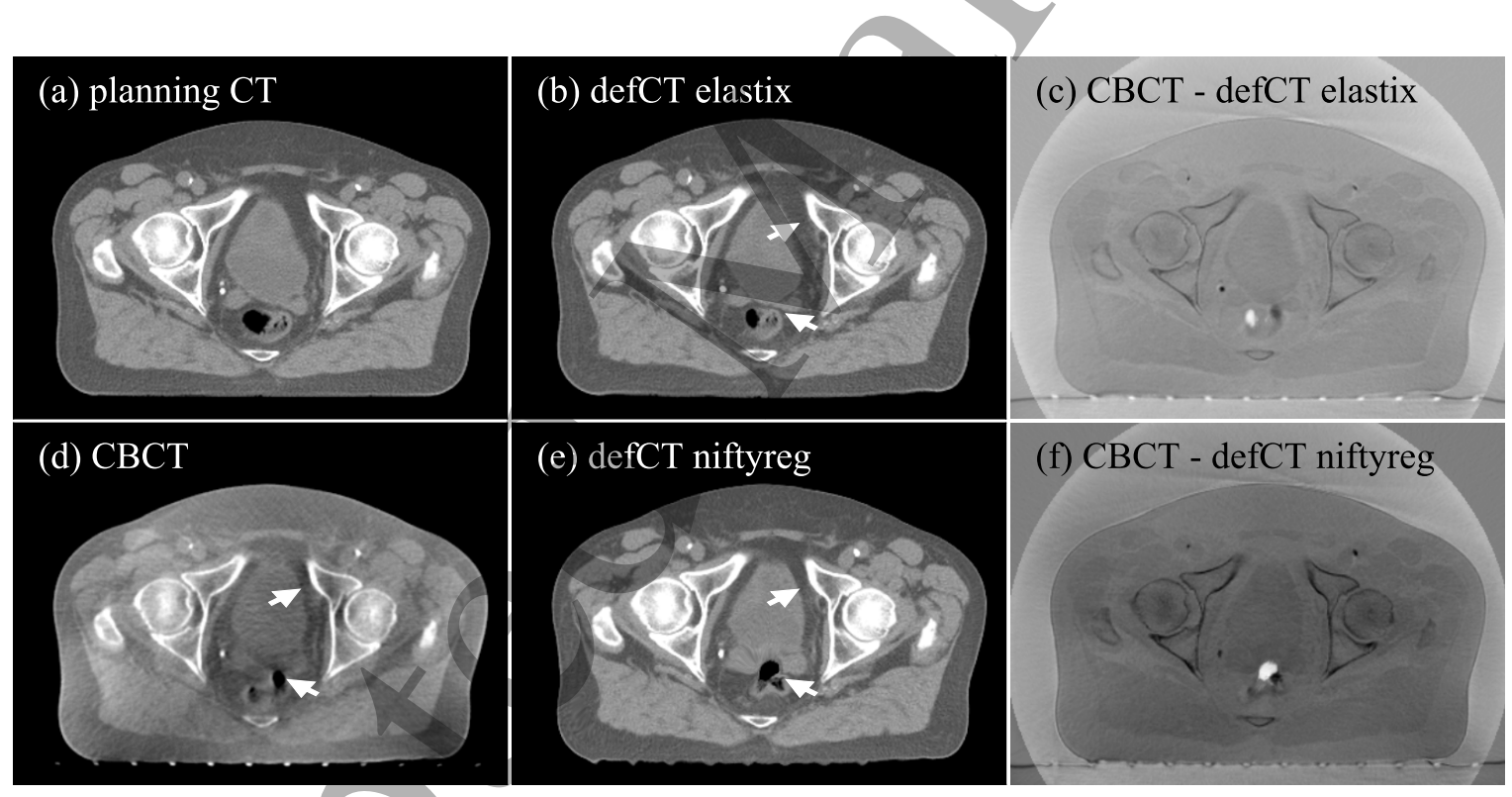

Figure 6. Example pelvis images demonstrating performance of DIR algorithms. (a) pCT image (b) Elastix deformed CT (c) subtraction of Elastix deformed CT from CBCT (d) original CBCT image (e) Niftyreg deformed CT (f) subtraction of Niftyreg deformed CT from CBCT.

Registration fails altogether for some CBCT images for both Elastix (3/132) and Niftyreg $(6 / 132)$. This was always due to failure of the initial rigid registration step. Usually this happens for images where there is a large difference in FOV between the CT and CBCT so the default alignment of image centres does not produce a sufficient overlap between the images. In these cases manual adjustments to the registration parameters and/or the initial alignment position were required. Registration failures were easily recognised on visual inspection and appeared as obvious non-physical distortions of the deformed CT image. 

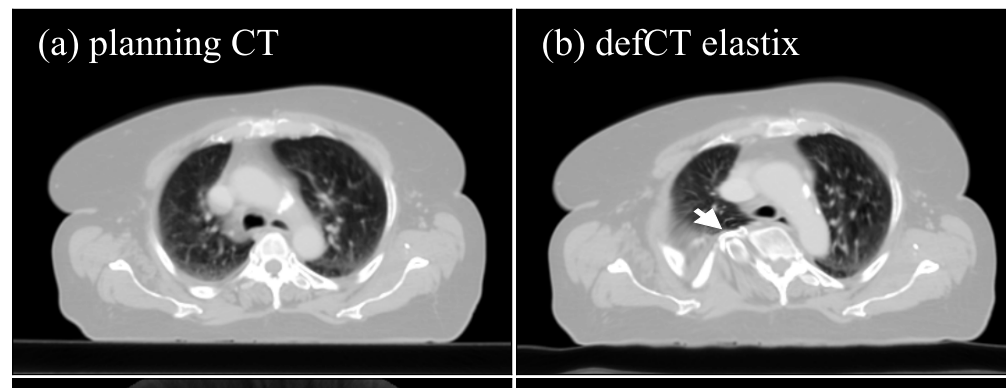

(c) CBCT - defCT elastix
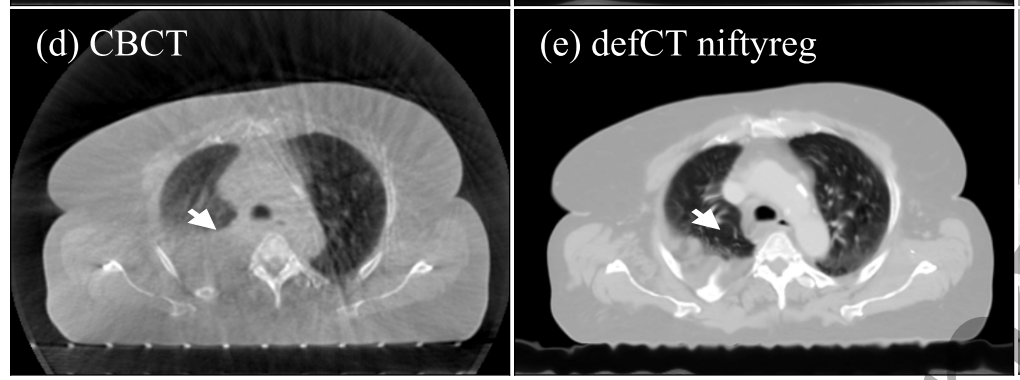

(f) CBCT - defCT niftyreg

Figure 7. Example lung images demonstrating performancé of DIR algorithms. (a) pCT image (b) Elastix deformed CT (c) subtraction of Elastix deformed CT from CBCT (d) original CBCT image (e) Niftyreg deformed CT (f) subtraction of Niftyreg deformed CT from CBCT.

\subsection{Pelvis images}

Figure 8 shows pelvis CBCT dose metric errors for raw CBCT, shading corrected CBCT and the two DIR algorithms. Raw CBCT shows the largest dose errors for all dose metrics with generally a large overestimation of dose and a large spread of errors. This is because the raw CBCT images have generally underestimated HU values, resulting in underestimated beam attenuation.

Shading corrected CBCT images show much smaller dose metric errors, with mean error less than $0.25 \%$ and standard deviation less than $0.6 \%$. No dose metrics had estimated errors greater than $2 \%$.

Both DIR algorithms resulted in similar dose metric error results. The mean dose metric error is less than $0.25 \%$ for all dose metrics studied for both Niftyreg and Elastix. Standard deviation of dose metric errors was also similar to shading corrected CBCT. This indicates that the soft-tissue mismatches in deformed CT noted in section section 3.3 do not give rise to large dose errors for pelvis images. However, both DIR algorithms led to a few images with dose metric error greater than $2 \%$ ( 2 for Niftyreg, 1 for Elastix).

\subsection{Lung images}

Figure 9 shows lung CBCT dose metric errors for raw CBCT, shading corrected CBCT and the two DIR algorithms. Raw CBCT results in the largest dose metric errors with mean error greater than $3 \%$ for all metrics and a large spread of errors.

Shading corrected CBCT images have much smaller dose metric errors, with mean error less than $0.5 \%$ and standard deviation of about $0.7 \%$. Dose metric error greater than $2 \%$ 


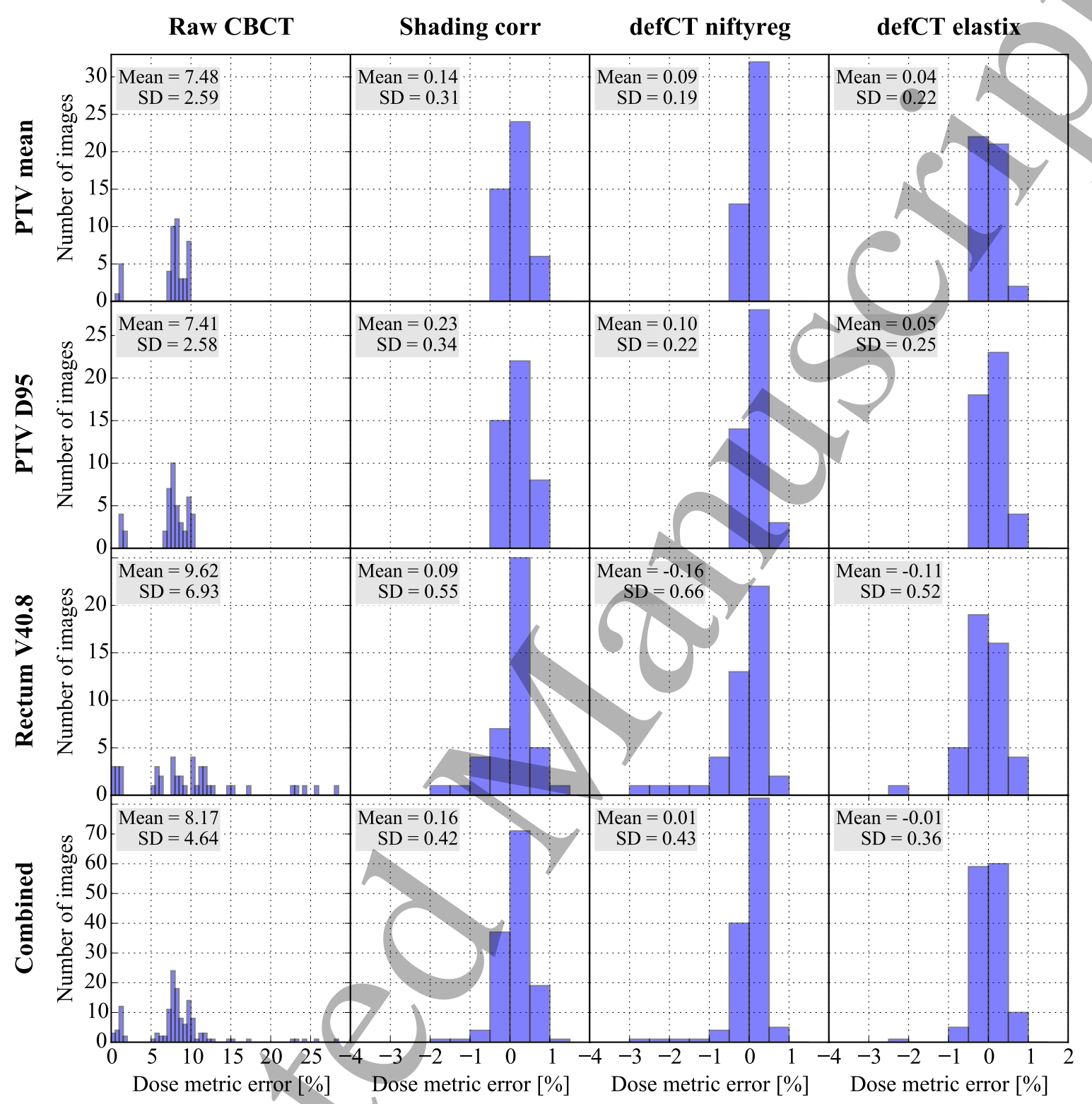

Figure 8. Dose metric errors for pelvis CBCT images. Columns 1,2,3 and 4 show results for raw CBCT, shading corrected CBCT, Niftyreg deformed CT and Elastix deformed CT respectively. Rows 1, 2, 3 and 4 show results for PTV mean dose, PTV $\mathrm{D}_{95}$, rectum $\mathrm{V}_{40 \mathrm{~Gy}}$ and all metrics combined respectively.

was observed in only one case.

Both DIR algorithms resulted in similar dose metric errors. The mean dose metric error was similar to shading corrected CBCT (less than $0.5 \%$ ), although the spread of errors was slightly larger than shading corrected CBCT (standard deivation about $1 \%$ ). Dose metric errors for deformed CT included more values greater than $2 \%$ (10 cases for both deformable registration algorithms). The images giving rise to these larger errors include cases such as illustrated in figure 7 with large changes between CT and CBCT which are not correctly represented by the deformed $\mathrm{CT}$. 
CBCT dose calculation accuracy comparison

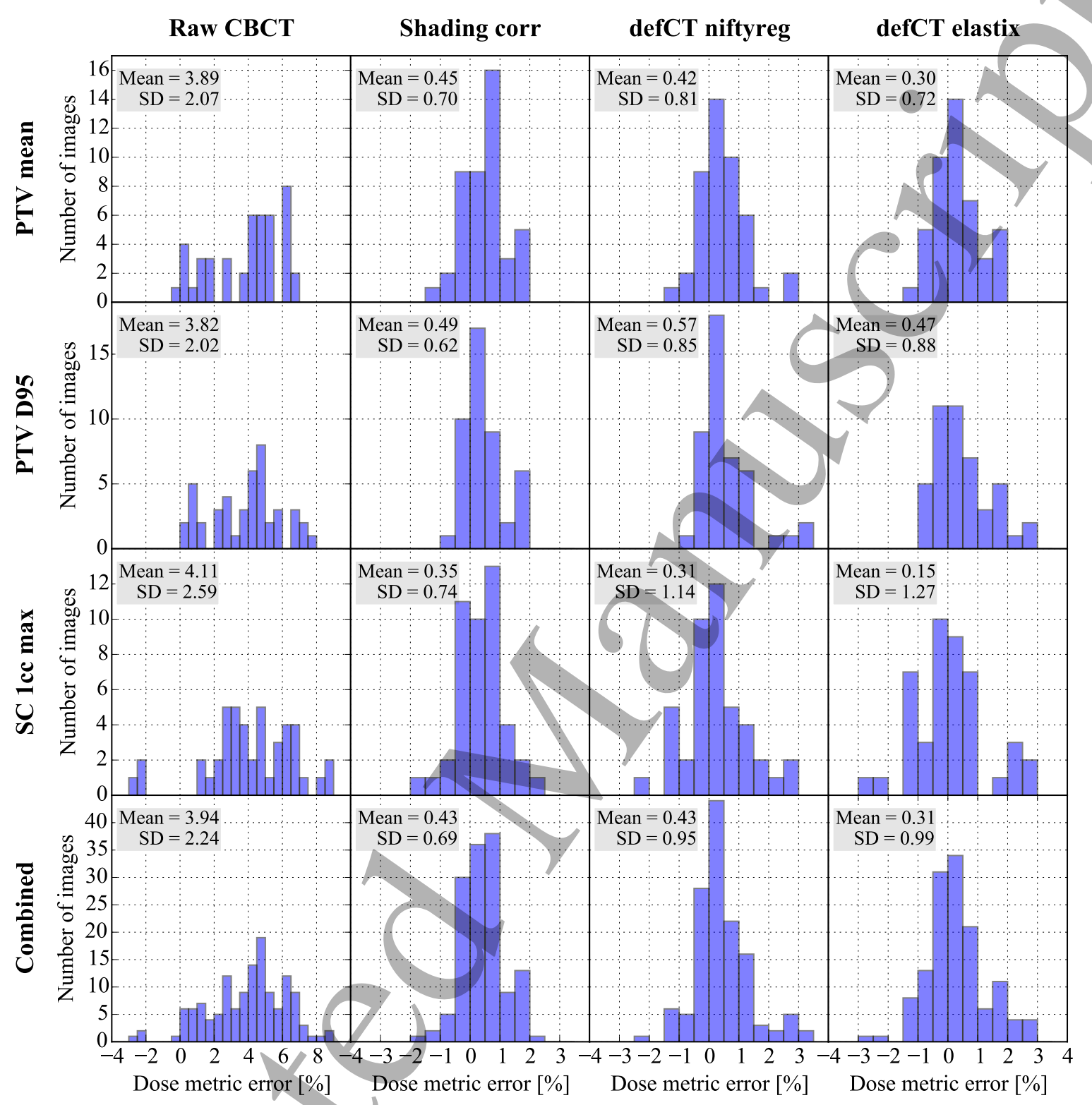

Figure 9. Dose metric errors for lung CBCT images. Columns 1,2,3 and 4 show results for raw CBCT, shading corrected CBCT, Niftyreg deformed CT and Elastix deformed CT respectively. Rows 1, 2, 3 and 4 show results for PTV mean dose, PTV D $_{95}$, SC 1cc max dose and all metrics combined respectively.

\subsection{Head and neck images}

Figure 10 shows CBCT dose metric errors assessed for H\&N patients for raw CBCT, shading corrected CBCT and the two DIR algorithms. Again the mean error and spread of errors is reduced for shading corrected CBCT and for both DIR algorithms. However for H\&N images the errors for raw CBCT are already quite small, so the improvement is not as large as for pelvis or lung images. See section 4 for further discussion of this. Shading corrected CBCT and both DIR methods all showed similar performance in terms of dose metric errors with overall mean error of $0.1 \%$ and standard deviation $0.4 \%$. No errors greater than $2 \%$ were 
CBCT dose calculation accuracy comparison

observed for any of the methods.

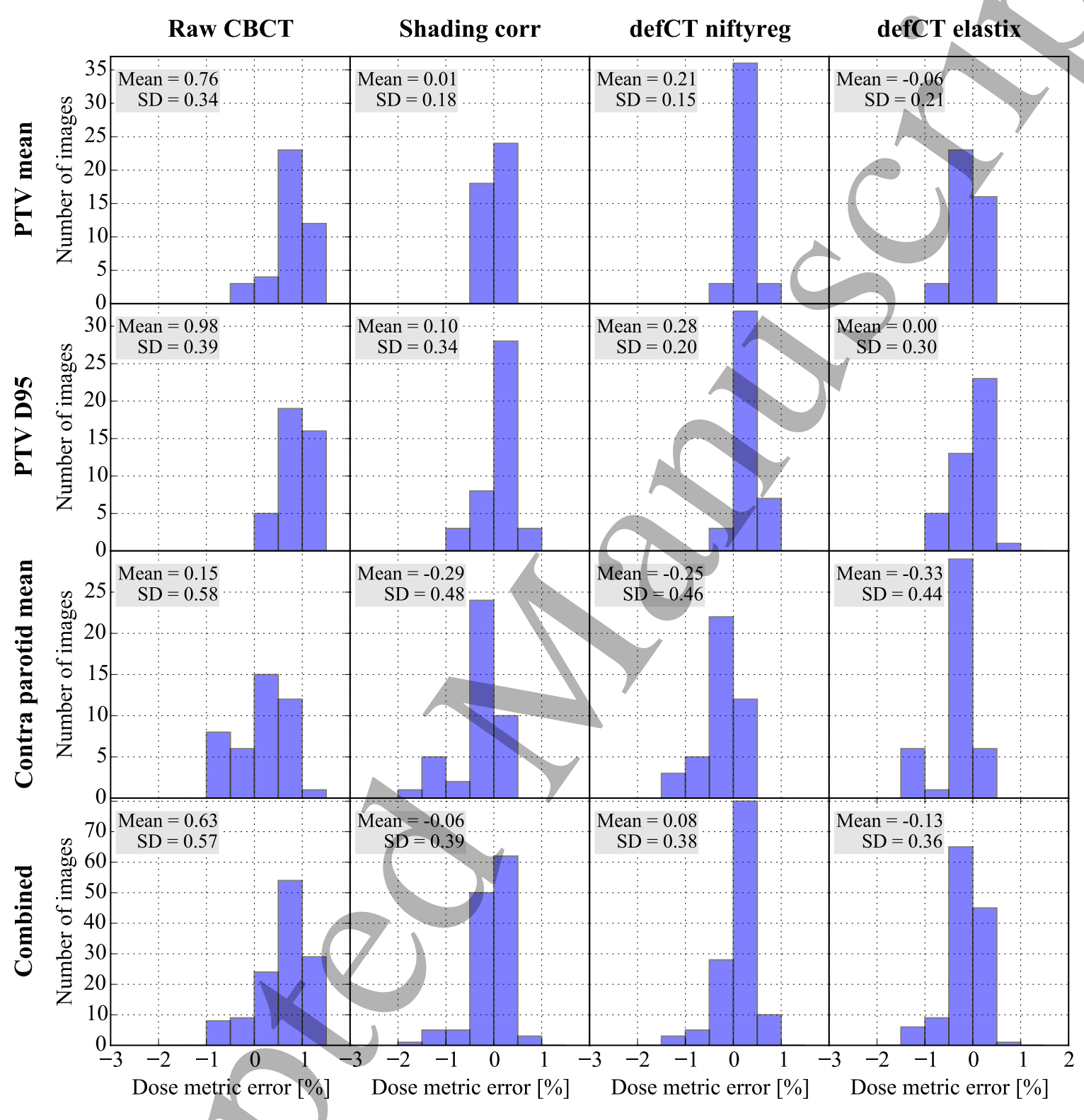

Figure 10. Dose metric errors for head and neck CBCT images. Columns 1,2,3 and 4 show results for raw CBCT, shading corrected CBCT, Niftyreg deformed CT and Elastix deformed CT respectively. Rows 1, 2, 3 and 4 show results for PTV mean dose, PTV $\mathrm{D}_{95}$, contralateral parotid mean dose and all metrics combined respectively.

\section{Discussion}

The results presented here indicate that RT dose calculations based on CBCT images can be assessed with relatively small errors (generally $<2 \%$ ) using any of the three methods tested (shading corrected CBCT, deformed CT with Elastix or Niftyreg). There is some evidence that the use of deformed CT leads to a larger spread of errors for lung and pelvis sites, with 


\section{CBCT dose calculation accuracy comparison}

more cases where the dose metric error was greater than $2 \%$. This occurred in $1-2 \%$ of cases for pelvis and $7 \%$ of cases for lung. We attribute these larger errors for calculations based on defomed CT to discrepancies between the CBCT and deformed CT anatomy in areas where large or complex anatomical changes have occurred. It may be possible to improve the performance of deformable registration through further optimization of parameters, however trade-offs will be required and it is a significant challenge to find parameters that will work robustly in all situations. We found that manual adjustment of initial image alignment was necessary in a number of cases. Thus the DIR algorithms cannot be considered to be fully automatic and a degree of manual supervision will likely be required. In contrast the shading correction operated without user intervention for all of the test images used in this study.

Dose metric errors for raw H\&N CBCT images (see figure 10) are generally smaller than those for raw pelvis or lung CBCT. This is explained firstly because the diameter of the head is smaller than either thorax or pelvis, causing fewer scattered X-rays with less severe scatter artefacts as a result. Secondly the majority of $H \& N$ images in our study were acquired with a newer version of the XVI software (v5.0) than for pelvis and lung images (mostly XVI v4.5). XVI v5.0 has improvements to calibration of CBCT Hounsfield Units, resulting in generally smaller dose metric errors, arguably good enough for clinical use without further correction. However there is still an improvement for shading corrected images or using DIR with pCT. This is because the changes in XVI v5.0 only improve the global normalization of the image, without correcting variations between different areas within the same image. It should also be noted that H\&N CBCT images feature severe artefacts below the level of the shoulders, also present in XVI v5.0 images, which are reduced by the shading correction (Joshi et al 2017). The effect of these artefacts is not seen in the dose errors presented here because we did not analyse dose in regions of the image where the patient contour was outside the FOV.

A number of other studies haves evaluated the accuracy of CBCT dose calculations using either image correction or deformed CT as the basis for attenuation correction. Dunlop et al. (Dunlop et al 2015) assessed CBCT dose calculation accuracy using either density overrides applied to the CBCT or scatter-corrected CBCT reconstructions. pCT acquired on the same day as the CBCT was used as ground truth. Based on a study of 11 patients they observed dose metric errors up to $4.0 \%$ for density override based methods. Scatter corrected CBCT was effective for H\&N images (errors up to $1.7 \%$ ) but less so for pelvis (errors up to $4.2 \%$ ) and lung (errors up to $8.0 \%$ ).

Onozato et al. (Onozato et al 2014) studied CBCT dose calculation accuracy for 10 prostate patients using two HU modification schemes (histogram matching to pCT or multi-level threshold segmentation into air/fat/muscle/bone). DIR to pCT was used to allow direct comparison of CBCT-based dose metrics to those calculated from the pCT. Both HU modification schemes produced small dose metric differences $(<2 \%)$ compared to the pCT.

Veiga et al. (Veiga et al 2014) assessed CBCT dose calculations based on deformed CT using Niftyreg. The study uses $5 \mathrm{H} \& \mathrm{~N}$ patients (1 CBCT per patient) and assessed dose calculation accuracy by comparison to repeat pCT taken close in time to the CBCT. Dose difference of $<2 \%$ was found in $90 \%$ of voxels within the body.

An notable feature of our study is the relatively large number of patients/images included 
(132 CBCTs in total). This is important because the dose metric errors typically form a normal distribution, which requires sufficient data points to get a good estimate of the width. With too little data we cannot reliably predict the frequency of large dose errors.

A limitation of our study is that the true CBCT dose distribution is unknown, meaning that we had to approximate it using the override-ratio method described in section 2.4.3. Some uncertainty in the estimates of dose metric error remains (as illustrated by our validation study where errors up to $1.7 \%$ were seen for patients with repeat CT imaging). Alternative validation methods have been used in other studies, e.g. comparison to CT image acquired on the same day (Dunlop et al 2015, Veiga et al 2014), or using DIR with pCT as ground truth (Onozato et al 2014). However these alternatives are also subject to uncertainties. There will be differences between images acquired on the same day, either due to transient anatomical changes or differences in patient set-up position. DIR could not be used as ground-truth in our study as it was one of the techniques under assessment.

While shading corrected CBCT provided similar or better dose accuracy to deformed CT and superior robustness in our study, there are some advantages to use of DIR in RT practice. The ability to use deformation fields to propogate contours onto CBCT or for dose accumulation is important to enable adaptive RT. However, the accuracy and robustness of DIR required for these purposes is higher than needed to use deformed CT for attenuation correction of RT dose calculations. For example the deformation errors illustrated in figure 6 did not have a significant effect on the RT dose distribution calculated using this image. However if organ contours propogated to CBCT via the deformation field were used then large errors in the estimated dose metrics could still occur due to incorrect estimation of the organ position. It is still an open question whether current DIR algorithms are good enough to support these uses (Li et al 2017, Schultheiss et al 2012).

A further obstacle to use of CBCT for calculation of radiotherapy dose distributions is the limited FOV. The FOV may not encompass all areas of the body traversed by the radiotherapy beams. Hence it will not be possible to calculate delivered dose unless a method is available to replace areas outside of the FOV. Possible methods include merging of multiple CBCTs to extend the FOV (Ding et al 2007), use of patient contour from rigidly registered pCT in areas outside the CBCT FOV (Dunlop et al 2015), and use of deformable registration with pCT to estimate patient contour in areas outside the CBCT FOV (Veiga et al 2014). In our study we only assessed dose in areas where the patient contour was fully within the CBCT FOV. Using medium or large FOV (41 cm or $50 \mathrm{~cm}$ diameter) this requirement was satisfied for the majority of pelvis and lung patients. Head and neck patients were usually imaged with small FOV (26 cm diameter), which was not large enough to cover the patient surface below the level of the shoulders. In these cases it may be necessary to image with larger FOV or to use one of the methods previously mentioned to replace the missing data.

\section{Conclusion}

Accuracy of CBCT-based RT dose calculations using either shading corrected CBCT or two DIR algorithms has been assessed in terms of clinically relevant target and OAR dose metrics 
for pelvis, lung and H\&N images. Similar performance is achieved by shading corrected CBCT and both DIR algorithms, with mean and standard deviation of dose metric error less than $1 \%$ for each of the sites studied. For lung images, use of deformed CT leads to slightly larger standard deviation of dose metric error than shading corrected CBCT with more dose metric errors greater than $2 \%$ observed ( $7 \%$ with deformed CT vs $1 \%$ with shading corrected CBCT). The results demonstrate that CBCT images processed using the shading correction algorithm or via deformable matching of pCT can be used to assess RT delivered dose with high confidence that dose metrics derived from the CBCT dose calculation will be accurate to within $2-3 \%$.

\section{Acknowledgments}

This work was supported by the UK Medical Research Council (MRC) grant No. MR/L023059/1.

\section{References}

Ding G, Duggan D, Coffey C, Deeley M, Hallahan D, Cmelak A and Malcolm A 2007 A study on adaptive IMRT treatment planning using kV cone-beam CT Radiotherapy and Oncology 85116

Dunlop A et al 2015 Comparison of CT number calibration techniques for CBCT-based dose calculation Strahlenther Onkol 191 970-8

Fan Q, Lu B, Park J C, Niu T, Li J G, Liu C and Zhu L 2015 Image-domain shading correction for cone-beam CT without prior patient information Journal of Applied Clinical Medical Physics 16 65-75

Fotina I, Hopfgartner J, StockM, Steininger T, Ltgendorf-Caucig C and Georg D 2012 Feasibility of CBCT-based dose calculation: Comparative analysis of HU adjustment techniques Radiotherapy and Oncology $104249-56$

Guan H and Dong H 2009 Dose calculation accuracy using cone-beam CT (CBCT) for pelvic adaptive radiotherapy Phys. Med. Biol. 546239

Jaffray D A, Siewerdsen J H, Wong J W and Martinez A A 2002 Flat-panel cone-beam computed tomography for image-guided radiation therapy International Journal of Radiation OncologyBiologyPhysics 53 1337-49

Joshi K D and Marchant T E 2017 Iterative peak combination: A robust technique for identifying relevant features in medical image histograms Biomed. Phys. Eng. Express In Press

Joshi K D, Marchant T E and Moore C J 2017 Shading correction algorithm for cone-beam CT in radiotherapy: extensive clinical validation of image quality improvement SPIE Medical Imaging 2017 Physics of Medical Imaging vol 10132 pp $101322 \mathrm{~A}-\mathrm{A}-13$

Kim J et al 2013 A novel approach for establishing benchmark CBCT/CT deformable image registrations in prostate cancer radiotherapy Phys. Med. Biol. 588077

Klein S, Staring M, Murphy K, Viergever M A and Pluim J P W 2010 elastix: A Toolbox for Intensity-Based Medical Image Registration IEEE Transactions on Medical Imaging 29 196-205

Li X, Zhang Y, Shi Y, Wu S, Xiao Y, Gu X, Zhen X and Zhou L 2017 Comprehensive evaluation of ten deformable image registration algorithms for contour propagation between CT and cone-beam CT images in adaptive head \& neck radiotherapy PLOS ONE 12 e0175906

Mail N, Moseley D J, Siewerdsen J H and Jaffray D A 2008 An empirical method for lag correction in cone-beam CT Med. Phys. 35 5187-96

Marchant T E, Joshi K D and Moore C J 2017 Shading correction for cone-beam CT in radiotherapy: validation of dose calculation accuracy using clinical images SPIE Medical Imaging 
2017 Physics of Medical Imaging vol 10132 pp 101320J-J-11

Marchant T E, Moore C J, Rowbottom C G, MacKay R I and Williams P C 2008 Shading correction algorithm for improvement of cone-beam CT images in radiotherapy Phys. Med. Biol. 535719

Marchant T E, Price G J, Matuszewski B J and Moore C J 2011 Reduction of motion artefacts in on-board cone beam CT by warping of projection images BJR $\mathbf{8 4} 251-64$

McBain C A et al $2006 \mathrm{X}$-ray volumetric imaging in image-guided radiotherapy: The new standard in on-treatment imaging International Journal of Radiation OncologyBiologyPhysics 64 62534

Modat M, Ridgway G R, Taylor Z A, Lehmann M, Barnes J, Hawkes D J, Fox N C and Ourselin S 2010 Fast free-form deformation using graphics processing units Computer Methods and Programs in Biomedicine 98 278-84

Moteabbed M, Sharp G C, Wang Y, Trofimov A, Efstathiou J A and Lu H-M 2015 Validation of a deformable image registration technique for cone beam CT-based dose verification Med. Phys. 42 196-205

Nithiananthan S et al 2011 Demons deformable registration of CT/and cone-beam CT using an iterative intensity matching approach Med. Phys. 38 1785-98

Niu T, Sun M, Star-Lack J, Gao H, Fan Q and Zhu L 2010 Shading correction for on-board cone-beam CT in radiation therapy using planning MDCT images Med. Phys. 37 5395-406

Onozato Y et al 2014 Evaluation of On-Board kV Cone Beam Computed TomographyBased Dose Calculation With Deformable Image Registration Using Hounsfield Unit Modifications International Journal of Radiation OncologyBiologyPhysics 89 416-23

Richter A, Hu Q, Steglich D, Baier K, Wilbert J, Guckenberger M and Flentje M 2008 Investigation of the usability of conebeam CT data sets for dose calculation Radiation Oncology 3 42

Rueckert D, Sonoda L I, Hayes C, Hill D L G, Leach M O and Hawkes D J 1999 Nonrigid registration using free-form deformations: application to breast MR images IEEE Transactions on Medical Imaging 18 712-21

Schultheiss T E, Tom W A and Orton C G 2012 It is not appropriate to "deform" dose along with deformable image registration in adaptive radiotherapy Med. Phys. 39 6531-3

Siewerdsen J H and Jaffray D A 2001 Cone-beam computed tomography with a flat-panel imager: Magnitude and effects of x-ray scatter Med. Phys. 28 220-31

Thing R S, Bernchou U, Mainegra-Hing E, Hansen O and Brink C 2016 Hounsfield unit recovery in clinical cone beam CT images of the thorax acquired for image guided radiation therapy Phys. Med. Biol. 615781

Veiga C, Alshaikhi J, Amos R, Loureno A M, Modat M, Ourselin S, Royle G and McClelland J R 2015 Cone-Beam Computed Tomography and Deformable Registration-Based "Dose of the Day" Calculations for Adaptive Proton Therapy International Journal of Particle Therapy 2 404-14

Veiga C et al 2014 Toward adaptive radiotherapy for head and neck patients: Feasibility study on using CT-to-CBCT deformable registration for "dose of the day" calculations Med. Phys. 41031703

Yang Y, Schreibmann E, Li T, Wang C and Xing L 2007 Evaluation of on-board kV cone beam CT (CBCT)-based dose calculation Phys. Med. Biol. 52685

Zhu L, Xie Y, Wang J and Xing L 2009 Scatter correction for cone-beam CT in radiation therapy Med. Phys. 36 2258-68 